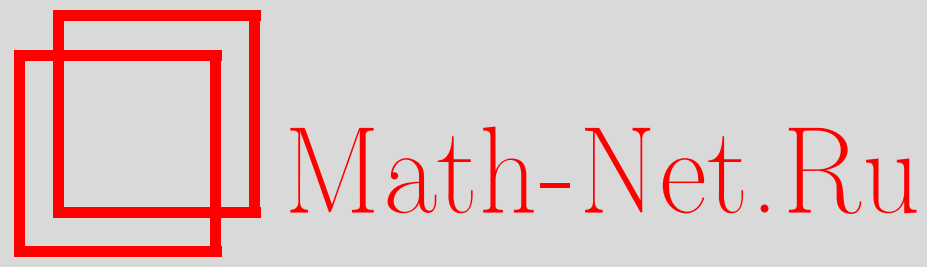

М. И. Исаев, Экспоненциальная неустойчивость в обратной задаче рассеяния на интервале энергий, Функи. анализ и его прил., 2013, том 47, выпуск $3,28-36$

DOI: https://doi.org/10.4213/faa3116

Использование Общероссийского математического портала MathNet.Ru подразумевает, что вы прочитали и согласны с пользовательским соглашением

http://www. mathnet.ru/rus/agreement

Параметры загрузки:

IP : 54.81 .137 .203

26 апреля 2023 г., 11:05:01

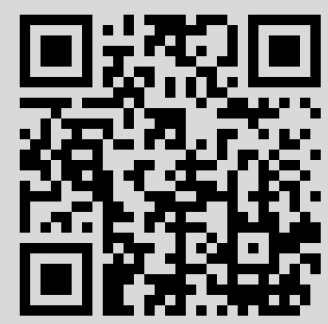


Функционалъный анализ и его приложения

2013, т. 47, вып. 3, с. 28-36

УДК 517.9

\title{
Экспоненциальная неустойчивость в обратной задаче рассеяния на интервале энергий
}

\author{
(C) 2013. М. И. ИСАЕВ
}

Рассмотрена трехмерная обратная задача рассеяния на интервале энергий. Работа посвящена вопросам устойчивости и неустойчивости в этой задаче. В частности, получены результаты об экспоненциальной неустойчивости, показывающие оптимальность с точностью до показателя в экспоненте логарифмической оценки, доказанной П. Стефановым в 1990 г. с использованием некоторой специальной нормы для амплитуды рассеяния при фиксированной энергии.

\section{$\S 1$. Введение}

Рассмотрим уравнение Шрёдингера

$$
-\Delta \psi+v(x) \psi=E \psi, \quad x \in \mathbb{R}^{3},
$$

где

$$
\begin{gathered}
\text { потенциал } v \text { является вещественным, } v \in L^{\infty}\left(\mathbb{R}^{3}\right), \\
v(x)=O\left(|x|^{-3-\varepsilon}\right) \text { при }|x| \rightarrow \infty \text { для некоторого } \varepsilon>0 .
\end{gathered}
$$

При выполнении условий (1.2) для любого $k \in \mathbb{R}^{3} \backslash 0$ уравнение (1.1) с $E=k^{2}$ имеет единственное непрерывное решение $\psi^{+}(x, k)$, имеющее асимптотику,

$$
\begin{gathered}
\psi^{+}(x, k)=e^{i k x}-2 \pi^{2} \frac{e^{i|k||x|}}{|x|} f\left(\frac{k}{|k|}, \frac{x}{|x|},|k|\right)+o\left(\frac{1}{|x|}\right) \\
\text { при }|x| \rightarrow \infty(\text { равномерно по } x /|x|),
\end{gathered}
$$

где $f(k /|k|, \omega,|k|)$ с фиксированным $k$ является непрерывной функцией от $\omega \in S^{2}$.

Функция $f(\theta, \omega, s)$, возникающая в $(1.3)$, называется амплитудой рассеяния для уравнения (1.1) с потенциалом $v$. (Дополнительную информацию о прямом рассеянии для уравнения (1.1) при выполнении условий (1.2) см., например, в [6] и [11].)

Хорошо известно, что для уравнения (1.1) при выполнении условий (1.2) амплитуда рассеяния $f$ в пределе высоких энергий однозначно определяет $\hat{v}$ на $\mathbb{R}^{3}$, где

$$
\hat{v}(p)=(2 \pi)^{-3} \int_{\mathbb{R}^{3}} e^{i p x} v(x) d x, \quad p \in \mathbb{R}^{3},
$$

через формулу Борна. В качестве математической теоремы этот результат был впервые приведен в [5] (подробную информацию см., например, в [11, разд. 2.1] и [14, теорема 1.1]).

Рассмотрим следующую обратную задачу для уравнения (1.1).

Задача 1.1. Дана амплитуда рассеяния $f$ на интервале энергий $I$. Найти $v$. 
В [7] показано, что для уравнения (1.1) при выполнении условий (1.2) для любого $E>0$ и $\delta>0$ амплитуда рассеяния $f(\theta, \omega, s)$ на $\left\{(\theta, \omega, s) \in S^{2} \times S^{2} \times R_{+}\right.$, $\left.E \leqslant s^{2} \leqslant E+\delta\right\}$ однозначно определяет $\hat{v}(p)$ на $\left\{p \in \mathbb{R}^{3}|| p \mid \leqslant 2 \sqrt{E}\right\}$. Доказательство основано на решении линейных интегральных уравнений и на аналитическом продолжении. Приведенный результат из [7] впоследствии был улучшен в [14]. С другой стороны, если $v$ удовлетворяет условиям (1.2), а также имеет компактный носитель или экспоненциально убывает на бесконечности, то $\hat{v}(p)$ на $\left\{p \in \mathbb{R}^{3}|| p \mid \leqslant 2 \sqrt{E}\right\}$ однозначно определяет $\hat{v}(p)$ на $\left\{p \in \mathbb{R}^{3}|| p \mid>2 \sqrt{E}\right\}$ посредством аналитического продолжения и, следовательно, однозначно определяет $v$ на $\mathbb{R}^{3}$.

В случае фиксированной энергии и потенциала $v$, удовлетворяющего условиям (1.2), а также имеющего компактный носитель или экспоненциально убывающего на бесконечности, результаты о глобальной единственности и точном восстановлении впервые получены в [12], [13].

Приближенный, но численно эффективный метод для поиска потенциала $v$ по амплитуде рассеяния $f$ в случае фиксированной энергии разработан в [15]. Результаты численной реализации этого метода представлены в [2].

Глобальные оценки устойчивости для задачи 1.1 получены Стефановым в [17] (для фиксированной энергии и потенциалов с компактным носителем), см. теорему 2.1 в 22 настоящей работы. С использованием специальной нормы для амплитуды рассеяния $f$ в [17] показано, что оценки устойчивости для задачи 1.1 следуют из оценок устойчивости Алессандрини [1] для обратной задачи Гельфанда-Кальдерона, т. е. задачи определения потенциала $v$ в ограниченной области по заданному оператору Дирихле-Неймана. Оценки устойчивости Алессандрини были недавно улучшены Новиковым [16].

В случае фиксированной энергии результаты Мандаша [10] доказывают, что оценки устойчивости Алессандрини и особенно Новикова оптимальны (с точностью до показателя экспоненты). В [8] исследования Мандаша были распространены на случай оператора Дирихле-Неймана, заданного на энергетических интервалах. Отметим, что оценки, подобные оценкам Мандаша, для эллиптических обратных задач, касающихся определения дефектов различного типа с помощью оператора Дирихле-Неймана или данных рассеяния, получены в [3], где была предложена также общая схема исследования вопросов неустойчивости такого типа. Хотя основной результат настоящей работы может быть представлен в рамках общей схемы из [3], это не приводит к заметному упрощению его полного доказательства.

В настоящей работе к задаче 1.1 применен подход, изложенный в [10], [3], [8], и показано, что логарифмические оценки Стефанова из [17] оптимальны (с точностью до показателя экспоненты). В $§ 2$ приведены оценки устойчивости из [17] (см. теорему 2.1) и результат настоящей работы о неустойчивости в задаче 1.1, см. теорему 2.2 и замечание 2.3. В $\S 3$ изложены доказательства некоторых основных аналитических свойств амплитуды рассеяния. В $\S 5$, развивая подход из [10], [3], [8], мы доказываем основной результат, теорему 2.2, используя оценки $\varepsilon$-энтропии и $\varepsilon$-емкости подходящих пространств, рассмотренных в $\S 4$.

\section{§2. Оценки устойчивости и неустойчивости}

В дальнейшем мы предполагаем, что

$$
\operatorname{supp} v(x) \subset D=B(0,1),
$$


где $B(x, r)$ обозначает открытый шар радиуса $r$ с центром $x$. Рассмотрим ортонормированный базис в $L^{2}\left(S^{2}\right)=L^{2}(\partial D)$ сферических гармоник

$$
\left\{Y_{j}^{p}: j \geqslant 0,1 \leqslant p \leqslant 2 j+1\right\} .
$$

Используя обозначение $a_{j_{1} p_{1} j_{2} p_{2}}$, мы будем подразумевать, что

$$
0 \leqslant j_{1}, \quad 1 \leqslant p_{1} \leqslant 2 j_{1}+1, \quad 0 \leqslant j_{2}, \quad 1 \leqslant p_{2} \leqslant 2 j_{2}+1 .
$$

Разложим функцию $f(\theta, \omega, s)$ в базисе $\left\{Y_{j_{1}}^{p_{1}} \times Y_{j_{2}}^{p_{2}}\right\}$ :

$$
f(\theta, \omega, s)=\sum_{j_{1}, p_{1}, j_{2}, p_{2}} a_{j_{1} p_{1} j_{2} p_{2}}(s) Y_{j_{1}}^{p_{1}}(\theta) Y_{j_{2}}^{p_{2}}(\omega) .
$$

Как и в [17], мы используем норму

$$
\|f(\cdot, \cdot, s)\|_{\sigma_{1}, \sigma_{2}}=\left\{\sum_{j_{1}, p_{1}, j_{2}, p_{2}}\left(\frac{2 j_{1}+1}{e s}\right)^{2 j_{1}+2 \sigma_{1}}\left(\frac{2 j_{2}+1}{e s}\right)^{2 j_{2}+2 \sigma_{2}}\left|a_{j_{1} p_{1} j_{2} p_{2}}(s)\right|^{2}\right\}^{1 / 2} .
$$

Если функция $f$ является амплитудой рассеяния для некоторого потенциала $v \in L^{\infty}(D)$ с носителем в $B(0, \rho)$, где $0<\rho<1$, то

$$
\left|a_{j_{1} p_{1} j_{2} p_{2}}(s)\right| \leqslant C\left(s,\|v\|_{L^{\infty}(D)}\right)\left(\frac{e s \rho}{2 j_{1}+1}\right)^{j_{1}+3 / 2}\left(\frac{e s \rho}{2 j_{2}+1}\right)^{j_{2}+3 / 2}
$$

и, следовательно, $\|f(\cdot, \cdot, s)\|_{\sigma_{1}, \sigma_{2}}<\infty$; см. оценки предложения 2.2 в [17].

Теорема 2.1 (см. [17]). Пусть вещественные потенциалы $v_{1}, v_{2}$ таковы, что $v_{i} \in L^{\infty}(D) \cap H^{q}\left(\mathbb{R}^{3}\right), \operatorname{supp} v_{i} \subset B(0, \rho),\left\|v_{i}\right\|_{L^{\infty}(D)} \leqslant N$ для $i=1,2 u$ некоторых $N>0, q>3 / 2$ и $0<\rho<1$. Пусть $f_{1} u f_{2}$ обозначают амплитуды рассеяния для уравнения (1.1) $с E=s^{2}, s>0$, п потенииалами $v_{1} u v_{2}$ соответственно. Тогда

$$
\left\|v_{1}-v_{2}\right\|_{L^{\infty}(D)} \leqslant c(N, \rho) \phi_{\delta}\left(\left\|f_{1}(\cdot, \cdot, s)-f_{2}(\cdot, \cdot, s)\right\|_{3 / 2,-1 / 2}\right),
$$

где норма $\|\cdot\|_{3 / 2,-1 / 2}$ определена в $(2.2)$, а $\phi_{\delta}(t)=(-\ln t)^{-\delta}$ для достаточно малых $t>0$ и некоторого фиксированного $\delta$, где, в частности, $0<\delta<1$.

Теорема 2.2. Для произвольного интервала $I=\left[s_{1}, s_{2}\right]$, где $s_{1}>0$, и для произвольных $m>0, \delta>2 m$ и действительных $\sigma_{1}, \sigma_{2}$ существуют константы $\beta>0$ и $N>0$, такие, что для любого $v_{0} \in C^{m}(D) c\left\|v_{0}\right\|_{L^{\infty}(D)} \leqslant N$ $u \operatorname{supp} v_{0} \subset B(0,1 / 2)$ и любого $\varepsilon \in(0, N)$ существуют потенииаль $v_{1}, v_{2} \in$ $C^{m}(D)$ с носителем в $B(0,1 / 2)$, удовлетворяющие условиям

$$
\begin{gathered}
\sup _{s \in I}\left(\left\|f_{1}(\cdot, \cdot, s)-f_{2}(\cdot, \cdot, s)\right\|_{\sigma_{1}, \sigma_{2}}\right) \leqslant \exp \left(-\varepsilon^{-1 / \delta}\right), \\
\left\|v_{1}-v_{2}\right\|_{L^{\infty}(D)} \geqslant \varepsilon, \\
\left\|v_{i}-v_{0}\right\|_{L^{\infty}(D)} \leqslant \varepsilon, \quad i=1,2, \quad\left\|v_{i}-v_{0}\right\|_{C^{m}(D)} \leqslant \beta, \quad i=1,2,
\end{gathered}
$$

где $f_{1}$ и $f_{2}$ обозначают амплитудь рассеяния для уравнения (1.1) с $E=s^{2}$, $s>0$, и потенциалами $v_{1}$ и $v_{2}$ соответственно.

Замечание 2.1. В случае фиксированной энергии $s_{1}=s_{2}$ можно заменить условие $\delta>2 m$ в теореме 2.2 на $\delta>5 m / 3$. 
Замечание 2.2. Можно считать $\beta$ в теореме 2.2 произвольно малым, если для некоторых констант $\varepsilon_{0}>0$ и $c>0$, зависящих от $\beta$, потребовать, чтобы $\varepsilon \leqslant \varepsilon_{0}$, и заменить правую часть в первом неравенстве в $(2.5)$ на $\exp \left(-c \varepsilon^{-1 / \delta}\right)$.

Замечание 2.3. Заметим, что в теореме 2.2 и замечании 2.1 подразумевается, в частности, что для любых вещественных $\sigma_{1}$ и $\sigma_{2}$ оценка

$$
\left\|v_{1}-v_{2}\right\|_{L^{\infty}(D)} \leqslant \tilde{c}(N, \rho, m, I) \sup _{s \in I} \phi_{\delta}\left(\left\|f_{1}(\cdot, \cdot, s)-f_{2}(\cdot, \cdot, s)\right\|_{\sigma_{1}, \sigma_{2}}\right)
$$

неверна в общем случае при $\delta>2 m$, если амплитуда рассеяния известна на интервале энергий, и при $\delta>5 \mathrm{~m} / 3$ для случая фиксированной энергии. Следовательно, из теоремы 2.2 и замечания 2.1 следует оптимальность логарифмической оценки устойчивости Стефанова (с точностью до показателя экспоненты).

Замечание 2.4. Недостатком оценки (2.4) является то, что $\delta<1$, даже если $m \rightarrow \infty$. По-видимому, исходя из результатов [16], оценку (2.4) можно улучшить до $\delta=m+o(m)$ при $m \rightarrow \infty$.

\section{§ 3. Некоторые основные аналитические свойства амплитуды рассеяния}

Рассмотрим решение $\psi^{+}(x, k)$ уравнения (1.1), см. формулу (1.3). Известно, что

$$
\psi^{+}(x, k)=e^{i k x} \mu^{+}(x, \theta, s),
$$

где $\theta \in S^{2}, k=s \theta$ и $\mu^{+}(x, \theta, s)$ является решением уравнения

$$
\begin{gathered}
\mu^{+}(x, \theta, s)=1-\int_{\mathbb{R}^{3}} G^{+}(x, y, s) e^{-i s \theta(x-y)} v(y) \mu^{+}(y, \theta, s) d y, \\
G^{+}(x, y, s)=\frac{e^{i s|x-y|}}{4 \pi|x-y|} .
\end{gathered}
$$

Мы полагаем, что условия (2.1) выполнены, а также что для некоторого $h>0$

$$
\begin{gathered}
|\operatorname{Im} s| \leqslant h, \\
c_{1}(h, D)\|v\|_{L^{\infty}(D)} \leqslant 1 / 2, \quad D=B(0,1), \\
c_{1}(h, D)=\sup _{x \in D} \int_{D} \frac{e^{2 h|x-y|}}{4 \pi|x-y|} d y .
\end{gathered}
$$

Тогда, в частности,

$$
\left|e^{-i s \theta(x-y)} e^{i s|x-y|}\right| \leqslant e^{2 h|x-y|} .
$$

Решая (3.1) методом последовательных приближений в $L^{\infty}(D)$, получаем, что

$$
\left|\mu^{+}(x, \theta, s)\right| \leqslant \frac{1}{1-c_{1}\|v\|_{L^{\infty}(D)}}, \quad \theta \in S^{2}, x \in D .
$$

Лемма 3.1. Пусть $f(\theta, \omega, s)$ - амплитуда рассеяния для потенииала $v \in$ $L^{\infty}(D)$, такого, что выполнены условия (2.1) и (3.3) для некоторого $h>0$, причем

$$
f(\theta, \omega, s)=\sum_{j_{1}, p_{1}, j_{2}, p_{2}} a_{j_{1} p_{1} j_{2} p_{2}}(s) Y_{j_{1}}^{p_{1}}(\theta) Y_{j_{2}}^{p_{2}}(\omega)
$$


- ее разложсение в базисе сферических гармоник $\left\{Y_{j_{1}}^{p_{1}} \times Y_{j_{2}}^{p_{2}}\right\}$. Тогда $a_{j_{1} p_{1} j_{2} p_{2}}(s)$ является голоморфной функиией в $W_{h}=\{s|| \operatorname{Im} s \mid \leqslant h\} u$

$$
\left|a_{j_{1} p_{1} j_{2} p_{2}}(s)\right| \leqslant c_{2}(h, D), \quad s \in W_{h} .
$$

Доказательство. Мы используем хорошо известную формулу

$$
f(\theta, \omega, s)=\frac{1}{(2 \pi)^{3}} \int_{\mathbb{R}^{3}} e^{i s(\theta-\omega) x} v(x) \mu^{+}(x, \theta, s) d x .
$$

Учитывая, что $\theta, \omega \in S^{2}$, приходим к неравенству

$$
\left|e^{i s(\theta-\omega) x}\right| \leqslant e^{2|\operatorname{Im} s \| x|} .
$$

Комбинируя это вместе с (2.1), (3.3), (3.5) и (3.6), мы получаем, что

$$
|f(\theta, \omega, s)| \leqslant \tilde{c}_{2}(h, D) \quad \text { для } s \in W_{h} .
$$

Так как

$$
a_{j_{1} p_{1} j_{2} p_{2}}(s)=\int_{S^{2} \times S^{2}} f(\theta, \omega, s) Y_{j_{1}}^{p_{1}}(\theta) Y_{j_{2}}^{p_{2}}(\omega) d \theta d \omega,
$$

это доказывает лемму 3.1 .

\section{$\S 4$. Оценки $\varepsilon$-энтропии и $\varepsilon$-емкости метрических пространств}

Определение 4.1. Рассмотрим метрическое пространство ( $X$, dist) и $\varepsilon>0$. Множество $Y \subset X$ называется $\varepsilon$-сетъю для $X_{1} \subset X$, если для любого $x \in X_{1}$ существует $y \in Y$, такое, что $\operatorname{dist}(x, y) \leqslant \varepsilon$. Тогда число $\mathscr{H}_{\varepsilon}\left(X_{1}\right):=\log _{2} \min \{|Y| \mid$ $Y$ является $\varepsilon$-сетью для $\left.X_{1}\right\}$ называется $\varepsilon$-энтропией множества $X_{1}$.

Множество $Z \subset X$ называется $\varepsilon$-дискретным, если для любых различных $z_{1}, z_{2} \in Z$ выполняется неравенство $\operatorname{dist}\left(z_{1}, z_{2}\right) \geqslant \varepsilon$. Тогда число $\mathscr{C}_{\varepsilon}:=$ $\log _{2} \max \left\{|Z| \mid Z \subset X_{1}\right.$, где $Z$ является $\varepsilon$-дискретным $\}$ называется $\varepsilon$-емкостъю множества $X_{1}$.

Использование $\varepsilon$-энтропии и $\varepsilon$-емкости для определения свойств отображений метрических пространств восходит к Витушкину и Колмогорову (см. [9] и ссылки, содержащиеся там). Одним из замечательных приложений является 13-я проблема Гильберта (о представлении функции нескольких переменных в виде суперпозиции функций меньшего числа переменных). По существу, лемма 4.1 и лемма 4.2 являются частями теорем XIV и XVII соответственно из [9]. Они также сформулированы и доказаны в [10] и [8] соответственно.

Лемма 4.1. Пусть $d \geqslant 2 u m>0$. Для $\varepsilon, \beta>0$ рассмотрим вещественное метрическое пространство

$$
X_{m \varepsilon \beta}=\left\{v \in C^{m}\left(\mathbb{R}^{d}\right) \mid \operatorname{supp} v \subset B(0,1 / 2),\|v\|_{L^{\infty}\left(\mathbb{R}^{d}\right)} \leqslant \varepsilon,\|v\|_{C^{m}\left(\mathbb{R}^{d}\right)} \leqslant \beta\right\}
$$

c метрикой из $L^{\infty}$. Тогда существует $\mu>0$, такое, что для любого $\beta>0 u$ $\varepsilon \in(0, \mu \beta)$ существует $\varepsilon$-дискретное множество $Z \subset X_{m \varepsilon \beta}$ с не менее чем $\exp \left(2^{-d-1}(\mu \beta / \varepsilon)^{d / m}\right)$ элементами.

Лемма 4.2. Пусть

$$
W_{I, \gamma}=\left\{\frac{a+b}{2}+\frac{a-b}{2} \cos z|| \operatorname{Im} z \mid \leqslant \gamma\right\}
$$

- эллипс в $\mathbb{C}$, где $I$ - некоторый интервал $[a, b]$ в $\mathbb{R} u \gamma>0$. Тогда существует константа $\nu=\nu(C, \gamma)>0$, такая, что для любого $\delta \in\left(0, e^{-1}\right)$ для 
пространства функиий на I с $L^{\infty}$-нормой, имеющих голоморфное продолэсение на $W_{I, \gamma}$, ограниченное в $W_{I, \gamma}$ по модулю константой $C$, существует $\delta$-сеть с не более чем $\exp \left(\nu\left(\ln \delta^{-1}\right)^{2}\right)$ элементами.

Замечание 4.1. В случае $a=b$ рассмотрим множество

$$
Y=\frac{\delta}{2} \mathbb{Z} \cap[-C, C]+i \cdot \frac{\delta}{2} \mathbb{Z} \cap[-C, C] .
$$

Мы получаем $\delta$-сеть с не более чем $\exp \left(\nu \ln \delta^{-1}\right)$ элементами.

Для интервала $I=\left[s_{1}, s_{2}\right]$, такого, что $s_{1}>0$, и вещественных $\sigma_{1}, \sigma_{2}$ рассмотрим банахово пространство

$$
X_{I, \sigma_{1}, \sigma_{2}}=\left\{\left(a_{j_{1} p_{1} j_{2} p_{2}}(s)\right) \mid\left\|\left(a_{j_{1} p_{1} j_{2} p_{2}}(s)\right)\right\|_{X_{I, \sigma_{1}, \sigma_{2}}}<\infty\right\}
$$

где

$$
\left\|\left(a_{j_{1} p_{1} j_{2} p_{2}}(s)\right)\right\|_{X_{I, \sigma_{1}, \sigma_{2}}}=\sup _{\substack{j_{1}, p_{1}, j_{2}, p_{2} \\ s \in I}}\left(\left(\frac{2 j_{1}+1}{e s}\right)^{j_{1}+\sigma_{1}}\left(\frac{2 j_{2}+1}{e s}\right)^{j_{2}+\sigma_{2}}\left|a_{j_{1} p_{1} j_{2} p_{2}}(s)\right|\right) .
$$

Пусть $f$ является амплитудой рассеяния для некоторого потенциала $v \in L^{\infty}(D)$ с носителем в $B(0, \rho)$, где $0<\rho<1$. Мы отождествляем амплитуду рассеяния $f(s, \theta, \omega)$ с ее матрицей коэффициентов $\left(a_{j_{1} p_{1} j_{2} p_{2}}(s)\right)$ в базисе сферических гармоник $\left\{Y_{j_{1}}^{p_{1}} \times Y_{j_{2}}^{p_{2}}\right\}$. Заметим, что

$$
\sup _{s \in I}\|f(\cdot, \cdot, s)\|_{\sigma_{1}, \sigma_{2}} \leqslant c_{3}\left\|\left(a_{j_{1} p_{1} j_{2} p_{2}}(s)\right)\right\|_{X_{I, \tilde{\sigma}_{1}, \tilde{\sigma}_{2}}},
$$

где $\tilde{\sigma}_{1}-\sigma_{2}=\tilde{\sigma}_{2}-\sigma_{2}=3$ и $c_{3}=c_{3}(I)>1$. Неравенство (4.3) следует из определений $(2.2),(4.2)$, если взять $c_{3}>1$, такое, что

$$
\sum_{j_{1}, p_{1}, j_{2}, p_{2}}\left(\frac{2 j_{1}+1}{e s}\right)^{-3}\left(\frac{2 j_{2}+1}{e s}\right)^{-3}<c_{3} .
$$

Для $h>0$ обозначим через $\mathscr{A}_{h}$ множество матриц, соответствующих амплитудам рассеяния для потенциалов $v \in L^{\infty}(D)$ с носителями в $B(0,1 / 2)$, таких, что выполнено условие (3.3).

Лемма 4.3. Для произвольного $h>0$ и вещественных $\sigma_{1}, \sigma_{2}$ множество $\mathscr{A}_{h}$ принадлежит $X_{I, \sigma_{1}, \sigma_{2}}$. Кроме того, существует $\eta=\eta\left(I, h, \sigma_{1}, \sigma_{2}\right)>0$, такое, что для любого $\delta \in\left(0, e^{-1}\right)$ для $\mathscr{A}_{h}$ в $X_{I, \sigma_{1}, \sigma_{2}}$ существует $\delta$-сеть $Y$ c не более чем $\exp \left(\eta\left(\ln \delta^{-1}\right)^{6}\left(1+\ln \ln \delta^{-1}\right)^{2}\right)$ элементами.

Доказательство. Можно считать, что $\sigma_{1}, \sigma_{2} \geqslant 0$, так как утверждение в данном случае сильнее. Если функция $f$ является амплитудой рассеяния для некоторого потенциала $v \in L^{\infty}(D)$ с носителем в $B(0,1 / 2)$, то из $(2.3)$ вытекает, что

$$
\left(\frac{2 j_{1}+1}{e s}\right)^{j_{1}+\sigma_{1}}\left(\frac{2 j_{2}+1}{e s}\right)^{j_{2}+\sigma_{2}}\left|a_{j_{1} p_{1} j_{2} p_{2}}(s)\right| \leqslant c_{4} \frac{\left(2 j_{1}+1\right)^{\sigma_{1}}\left(2 j_{2}+1\right)^{\sigma_{2}}}{2^{j_{1}+j_{2}}}
$$

где $c_{4}=c_{4}(I, h)>0$. Следовательно, для любых положительных $\sigma_{1}$ и $\sigma_{2}$

$$
\left\|\left(a_{j_{1} p_{1} j_{2} p_{2}}(s)\right)\right\|_{X_{I, \sigma_{1}, \sigma_{2}}} \leqslant \sup _{j_{1}, j_{2}}\left(c_{4} \frac{\left(2 j_{1}+1\right)^{\sigma_{1}}\left(2 j_{2}+1\right)^{\sigma_{2}}}{2^{j_{1}+j_{2}}}\right)<\infty,
$$

а значит, первое утверждение леммы доказано. 
Пусть $l_{\delta, \sigma_{1}, \sigma_{2}}$ - наименьшее натуральное число, такое, что $c_{4}(2 l+1)^{\sigma_{1}+\sigma_{2}} 2^{-l}$ $<\delta$ для любого $l \geqslant l_{\delta, \sigma_{1}, \sigma_{2}}$. Взяв натуральный логарифм, находим, что

$$
-\ln c_{4}-\left(\sigma_{1}+\sigma_{2}\right) \ln (2 l+1)+l \ln 2>\ln \delta^{-1} \quad \text { при } l \geqslant l_{\delta, \sigma_{1}, \sigma_{2}} .
$$

Используя $\ln \delta^{-1}>1$, получаем, что

$$
l_{\delta, \sigma_{1}, \sigma_{2}} \leqslant C^{\prime} \ln \delta^{-1},
$$

где константа $C^{\prime}$ зависит только от $h, \sigma_{1}, \sigma_{2}$ и $I=\left[s_{1}, s_{2}\right]$. Положим $W_{I}=$ $W_{I, \gamma}$ (см. (4.1)), где константа $\gamma>0$ такова, что $W_{I} \subset\{s|| \operatorname{Im} s \mid \leqslant h\}$. Если $\max \left(j_{1}, j_{2}\right) \leqslant l_{\delta, \sigma_{1}, \sigma_{2}}$, то обозначим через $Y_{j_{1} p_{1} j_{2} p_{2}}$ некоторую $\delta_{j_{1} p_{1} j_{2} p_{2}}$-сеть из леммы 4.2 с константой $C=c_{2}$, где $c_{2}$ - константа из леммы 3.1 и

$$
\delta_{j_{1} p_{1} j_{2} p_{2}}=\left(\frac{e s_{1}}{2 j_{1}+1}\right)^{j_{1}+\sigma_{1}}\left(\frac{e s_{1}}{2 j_{2}+1}\right)^{j_{2}+\sigma_{2}} \delta .
$$

В противном случае положим $Y_{j_{1} p_{1} j_{2} p_{2}}=\{0\}$. Пусть

$$
Y=\left\{\left(a_{j_{1} p_{1} j_{2} p_{2}}(s)\right) \mid a_{j_{1} p_{1} j_{2} p_{2}}(s) \in Y_{j_{1} p_{1} j_{2} p_{2}}\right\} .
$$

Для любого $\left(a_{j_{1} p_{1} j_{2} p_{2}}(s)\right) \in \mathscr{A}_{h}$ существует элемент $\left(b_{j_{1} p_{1} j_{2} p_{2}}(s)\right) \in Y$, такой, что

$$
\begin{aligned}
&\left(\frac{2 j_{1}+1}{e s}\right)^{j_{1}+\sigma_{1}}\left(\frac{2 j_{2}+1}{e s}\right)^{j_{2}+\sigma_{2}}\left|a_{j_{1} p_{1} j_{2} p_{2}}(s)-b_{j_{1} p_{1} j_{2} p_{2}}(s)\right| \\
& \leqslant\left(\frac{2 j_{1}+1}{e s}\right)^{j_{1}+\sigma_{1}}\left(\frac{2 j_{2}+1}{e s}\right)^{j_{2}+\sigma_{2}} \delta_{j_{1} p_{1} j_{2} p_{2}} \leqslant \delta,
\end{aligned}
$$

если $\max \left(j_{1}, j_{2}\right) \leqslant l_{\delta, \sigma_{1}, \sigma_{2}}$, и

$$
\begin{aligned}
\left(\frac{2 j_{1}+1}{e s}\right)^{j_{1}+\sigma_{1}} & \left(\frac{2 j_{2}+1}{e s}\right)^{j_{2}+\sigma_{2}}\left|a_{j_{1} p_{1} j_{2} p_{2}}(s)-b_{j_{1} p_{1} j_{2} p_{2}}(s)\right| \\
& \leqslant c_{4} \frac{\left(2 j_{1}+1\right)^{\sigma_{1}}\left(2 j_{2}+1\right)^{\sigma_{2}}}{2^{j_{1}+j_{2}}} \leqslant c_{4} \frac{\left(2 \max \left(j_{1}, j_{2}\right)+1\right)^{\sigma_{1}+\sigma_{2}}}{2^{\max \left(j_{1}, j_{2}\right)}}<\delta
\end{aligned}
$$

в противном случае.

Найдем число элементов множества $Y$. Заметим, что $\left|Y_{j_{1} p_{1} j_{2} p_{2}}\right|=1$, если $\max \left(j_{1}, j_{2}\right)>l_{\delta, \sigma_{1}, \sigma_{2}}$. Используя еще раз неравенство $\ln \delta^{-1} \geqslant 1$ и (4.4), получаем следующую оценку в случае, когда $\max \left(j_{1}, j_{2}\right) \leqslant l_{\delta, \sigma_{1}, \sigma_{2}}$ :

$$
\left|Y_{j_{1} p_{1} j_{2} p_{2}}\right| \leqslant \exp \left(\nu\left(\ln \delta_{j_{1} p_{1} j_{2} p_{2}}^{-1}\right)^{2}\right) \leqslant \exp \left(\nu^{\prime}\left(\ln \delta^{-1}\right)^{2}\left(1+\ln \ln \delta^{-1}\right)^{2}\right) .
$$

Заметим, что $n_{\delta, \sigma_{1}, \sigma_{2}} \leqslant l_{\delta, \sigma_{1}, \sigma_{2}}^{2}\left(2 l_{\delta, \sigma_{1}, \sigma_{2}}+1\right)^{2} \leqslant\left(2 l_{\delta, \sigma_{1}, \sigma_{2}}+1\right)^{4}$, где $n_{\delta, \sigma_{1}, \sigma_{2}}$ обозначает число четверок $\left(j_{1}, p_{1}, j_{2}, p_{2}\right)$, таких, что $\max \left(j_{1}, j_{2}\right) \leqslant l_{\delta, \sigma_{1}, \sigma_{2}}$. Тогда существует достаточно большое $\eta$, такое, что

$$
\begin{aligned}
|Y| & \leqslant\left(\exp \left(\nu^{\prime}\left(\ln \delta^{-1}\right)^{2}\left(1+\ln \ln \delta^{-1}\right)^{2}\right)\right)^{n_{\delta, \sigma_{1}, \sigma_{2}}} \\
& \leqslant \exp \left(\nu^{\prime}\left(\ln \delta^{-1}\right)^{2}\left(1+\ln \ln \delta^{-1}\right)^{2}\left(1+2 C^{\prime} \ln \delta^{-1}\right)^{4}\right) \\
& \leqslant \exp \left(\eta\left(\ln \delta^{-1}\right)^{6}\left(1+\ln \ln \delta^{-1}\right)^{2}\right) .
\end{aligned}
$$

Замечание 4.2. В случае $s_{1}=s_{2}$, учитывая замечание 4.1 и используя его в $(4.5)$ и (4.6), мы получим $\delta$-сеть $Y$ с не более чем $\exp \left(\eta\left(\ln \delta^{-1}\right)^{5}\left(1+\ln \ln \delta^{-1}\right)\right)$ элементами. 


\section{§5. Доказательство теоремы 2.2}

Выберем $N$ таким образом, чтобы условие (3.3) выполнялось для любого $\|v\|_{L^{\infty}(D)} \leqslant 2 N$ и некоторого фиксированного $h>0$. Согласно лемме 4.1, множество $v_{0}+X_{m \varepsilon \beta}$ имеет $\varepsilon$-дискретное подмножество $v_{0}+Z$. Так как $\varepsilon \in(0, N)$, то множество $Y$, построенное в лемме 4.3 , является также $\delta$-сетью для множества матриц, соответствующих амплитудам рассеяния для потенциалов $v \in$ $v_{0}+X_{m \varepsilon \beta}$. Возьмем $\delta$, такое, что $2 c_{3} \delta=\exp \left(-\varepsilon^{-1 / \alpha}\right)$, см. (4.3). Заметим, что неравенства (2.5) следуют из неравенства

$$
\left|v_{0}+Z\right|>|Y|,
$$

где $Y$ обозначает множество, построенное в лемме 4.3 , с $\tilde{\sigma}_{1}=\sigma_{1}+3$ и $\tilde{\sigma}_{2}=$ $\sigma_{2}+3$. Действительно, если $\left|v_{0}+Z\right|>|Y|$, то существуют два потенциала $v_{1}, v_{2} \in v_{0}+Z$ с матрицами $\left(a_{j_{1} p_{1} j_{2} p_{2}}(s)\right)$ и $\left(b_{j_{1} p_{1} j_{2} p_{2}}(s)\right)$, соответствующими амплитудам рассеяния, которые находятся в одном $X_{I, \sigma_{1}, \sigma_{2}}$-шаре радиуса $\delta \mathrm{c}$ центром в элементе $Y$. Следовательно, используя (4.3), мы получаем, что

$$
\begin{aligned}
\sup _{s \in I}\left\|f_{1}(\cdot, \cdot, s)-f_{2}(\cdot, \cdot, s)\right\|_{\sigma_{1}, \sigma_{2}} & \leqslant c_{3}\left\|\left(a_{j_{1} p_{1} j_{2} p_{2}}(s)\right)-\left(b_{j_{1} p_{1} j_{2} p_{2}}(s)\right)\right\|_{X_{I, \sigma_{1}, \tilde{\sigma}_{2}}} \\
& \leqslant 2 c_{3} \delta=\exp \left(-\varepsilon^{-1 / \alpha}\right) .
\end{aligned}
$$

Найдем $\beta$, такое, чтобы условие (5.1) выполнялось. Используя лемму 4.3 , для некоторого $\eta_{\alpha}=\eta_{\alpha}\left(I, \sigma_{1}, \sigma_{2}, \alpha\right)>0$ получаем, что

$$
|Y| \leqslant \exp \left(\eta\left(\ln \left(2 c_{3}\right)+\varepsilon^{-1 / \alpha}\right)^{6}\left(1+\ln \left(\ln \left(2 c_{3}\right)+\varepsilon^{-1 / \alpha}\right)\right)^{2}\right) \leqslant \exp \left(\eta_{\alpha} \varepsilon^{-3 / m}\right) .
$$

Тогда положим

$$
\beta>\mu^{-1} \max \left(N, \eta_{\alpha}^{m / 3} 2^{2 m}\right) .
$$

Получаем, что условие $\varepsilon<\mu \beta$ в лемме 4.1 выполнено; следовательно,

$$
\left|v_{0}+Z\right|=|Z| \geqslant \exp \left(2^{-4}(\mu \beta / \varepsilon)^{3 / m}\right) \stackrel{(5.3)}{>} \exp \left(2^{-4}\left(\eta_{\alpha}^{m / 3} 2^{2 m} / \varepsilon\right)^{3 / m}\right) \stackrel{(5.2)}{\geqslant}|Y| .
$$

Последнее неравенство завершает доказательство теоремы 2.2.

В случае фиксированной энергии $s_{1}=s_{2}$, используя замечание 4.2 в (5.2), можно заменить условие $\alpha>2 m$ в теореме 2.2 на $\alpha>5 m / 3$.

$$
* * *
$$

Настоящая работа выполнена под руководством Р. Г. Новикова в рамках стажировки в Ecole Polytechnique.

\section{ЛитературА}

[1] G. Alessandrini, Stable determination of conductivity by boundary measurements, Appl. Anal., 27:1-3 (1988), 153-172.

[2] Н. В. Алексеенко, В. А. Буров, О. Д. Румянцева, Решение трехмерной обратной задачи акустического рассеяния II. Модифицированный алгоритм Новикова, Акустич. журнал, 54:3 (2008), 469-482.

[3] M. Di Cristo, L. Rondi, Examples of exponential instability for inverse inclusion and scattering problems, Inverse Problems, 19:3 (2003), 685-701.

[4] I. M. Gelfand, Some aspects of functional analysis and algebra, in: Proc. Internat. Congress of Math., Amsterdam, 1954, vol. 1, North Holland, Amsterdam, 1957, 253276.

[5] Л. Д. Фаддеев, Единственность решения обратной задачи рассеяния, Вестник Ленинградск. ун-та, 11:7 (1956), 126-130. 
[6] Л. Д. Фаддеев, Обратная задача квантовой теории рассеяния. II, в кн.: Итоги науки и техники. Современные проблемы математики, т. 3, ВИНИТИ, М., 1974, 93-180.

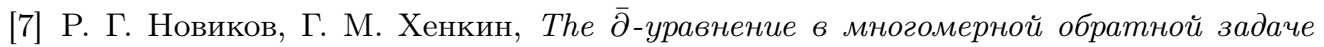
рассеяния, УМН, 42:3(255) (1987), 93-152.

[8] M. I. Isaev, Exponential instability in the Gel'fand inverse problem on the energy intervals, J. Inverse Ill-Posed Probl., 19:3 (2011), 453-473.

[9] А. Н. Колмогоров, В. М. Тихомиров, $\varepsilon$-энтропия и $\varepsilon$-емкость множеств в функциональных пространствах, УМН, 14:2(86) (1959), 3-86.

[10] N. Mandache, Exponential instability in an inverse problem for the Schrödinger equation, Inverse Problems, 17:5 (2001), 1435-1444.

[11] R. G. Newton, Inverse Schrodinger Scattering in Three Dimensions, Texts and Monographs in Physics, Springer-Verlag, Berlin, 1989.

[12] Р. Г. Новиков, Многомерная обратная спектральная задача для уравнения $-\Delta \psi+$ $(v(x)-E u(x)) \psi=0$, Функц. анализ и его прил., 22:4 (1988), 11-22.

[13] R. G. Novikov, The inverse scattering problem at fixed energy for the three-dimensional Schrödinger equation with an exponentially decreasing potential, Comm. Math. Phys., 161:3 (1994), 569-595.

[14] R. G. Novikov, On determination of the Fourier transform of a potential from the scattering amplitude, Inverse Problems, 17:5 (2001), 1243-1251.

[15] R. G. Novikov, The $\bar{\partial}$-approach to approximate inverse scattering at fixed energy in three dimensions, IMRP Int. Math. Res. Pap., 6 (2005), 287-349.

[16] R. G. Novikov, New global stability estimates for the Gelfand-Calderon inverse problem, Inverse Problems, 27:1 (2011), 015001.

[17] P. Stefanov, Stability of the inverse problem in potential scattering at fixed energy, Ann. Inst. Fourier (Grenoble), 40:4 (1990), 867-884.

Московский физико-технический институт (НИУ)

Centre de Mathématiques Appliquées, Ecole Polytechnique

Поступило в редакцию

e-mail: isaev.m.i@gmail.com

7 июня 2011 г. 